\section{Wie effektiv wirkt Psychotherapie bei ADHS - subjektiv und objektiv beurteilt?}

\section{Im subjektiven Empfinden von Patienten schneidet Gruppentherapie plus Medikation besser ab als objektive Beurteilungen mittels CGI-I und anderen Skalen vermuten lassen.}

G roß et al. veröffentlichten in diesem Jahr die Ergebnisse der COMPASStudie - einer multizentrischen Studie zum Vergleich von Methylphenidat (MPH) und Psychotherapie. Verglichen wurden vier Behandlungsgruppen: _Gruppenpsychotherapie plus MPH _ Gruppenpsychotherapie plus Placebo _Klinisches Management plus MPH -Klinisches Management plus Placebo Die 120-minütige Gruppentherapie (zweimal 50-Minuten-Einheiten mit 20 Minuten Pause dazwischen) wurde nach dem Manual von Hesslinger (2014) durchgeführt und basiert auf Behandlungsstrategien der DBT (dialektisch be- haviorale Therapie) nach Marsha Linehan und der KBT (kognitiv behaviorale Therapie). Sie wurde zwölf Wochen wöchentlich und im Anschluss zehn Wochen vierwöchentlich durchgeführt.

Das klinische Management bestand aus nondirektiver individueller psychiatrischer Beratung, die keiner spezifischen oder strukturiert behavioralen Intervention folgte. Die Einzelsitzungen dauerten 15 bis 20 Minuten und wurden ebenfalls für zwölf Wochen wöchentlich und im Anschluss für zehn Wochen monatlich durchgeführt.

Die medikamentöse Behandlung erfolgte doppelblind mit MPH oder Place-

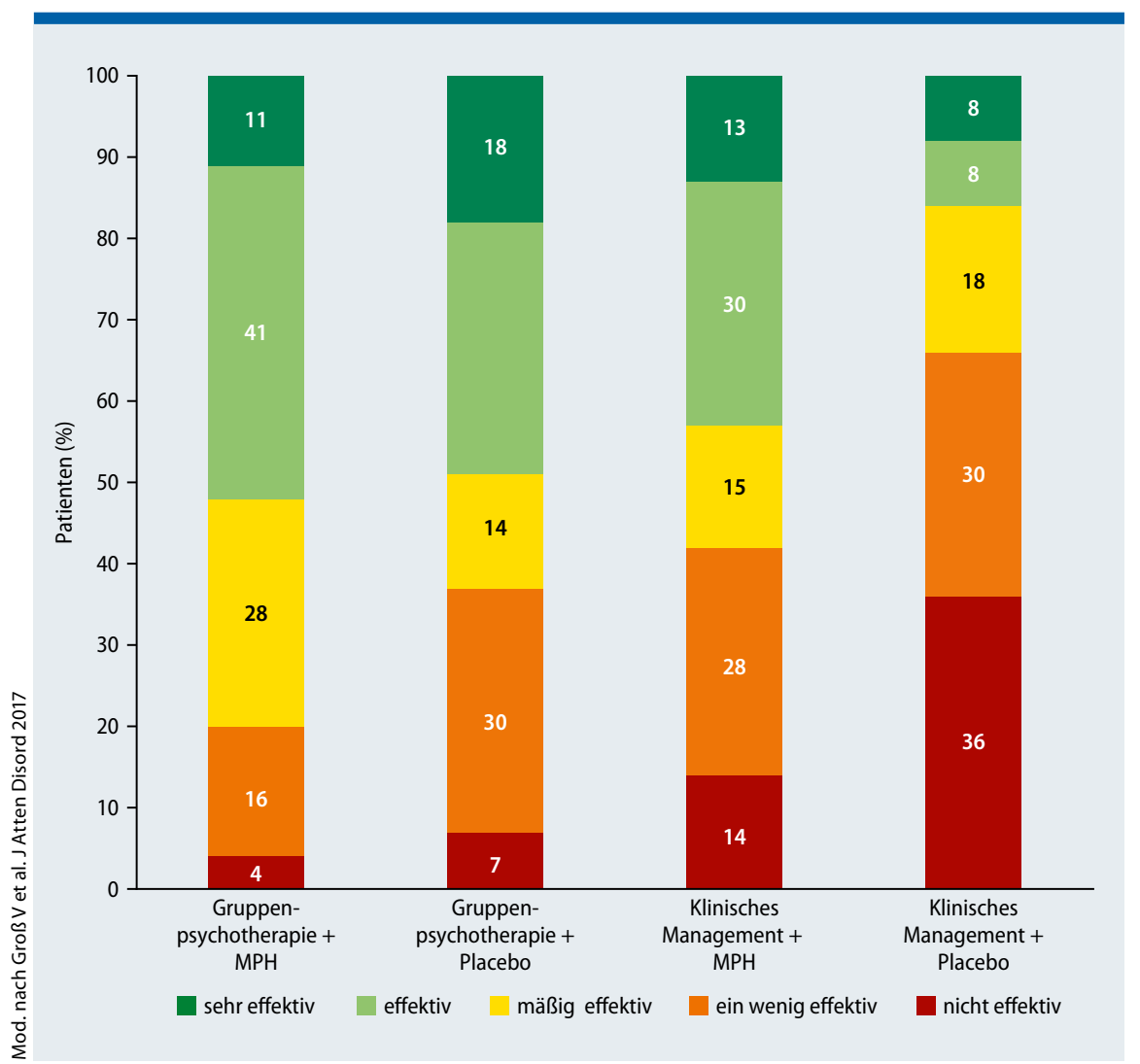

Abb. 3: Subjektive retrospektive Einschätzung der Effektivität der Behandlung durch den Patienten 52 Wochen nach Behandlungsstart. bo. Die Startdosis von MPH betrug 10 $\mathrm{mg} /$ Tag. Innerhalb von 52 Wochen wurde die Dosis individuell angepasst auf eine Maximaldosis von 1,3 mg/kg KG.

Die Bewertung erfolgte sowohl durch Selbst- (Patienten) als auch durch Fremdrating (qualifizierte Rater, „Observer").

Gruppentherapie plus MPH war in der Patientenbefragung den anderen Gruppen signifikant überlegen (Abb. 3). Am schlechtesten war die Zufriedenheit bei Patienten, die nur Einzelsitzungen und Placebo erhalten hatten. Die objektivere Beurteilung von „Observern“ mittels CGI-I (Clinical Global Impression of Improvement), CGA-E (Clinical Global Assessment of Effectivness) und CAARS-O:L (observer related interviews) konnte hingegen - gegen die Erwartungen des Konsortiums - keinen Unterschied zwischen Gruppentherapie und stützenden Einzelgesprächen jeweils plus MPH zeigen.

\section{Kommentar}

Diese Studie zeigt beeindruckend, wie sich das subjektive Empfinden von objektiveren Skalen unterscheiden kann. Aus Patientenperspektive erweist sich die Pharmakotherapie ohne begleitende Psychotherapie als wenig wirksam. Der Benefit für Patienten ergibt sich vor allem in Gruppentherapien, in denen sie geschult werden und lernen, mit der Erkrankung umzugehen. Sicher wirkt sich auch der Kontakt zur Peergroup insgesamt günstig auf das subjektive Erleben und die Verbesserung des Zustands, wie von den Patienten im Rating angegeben, aus.

Prof. Dr. med. Sibylle C. Roll, Prof. Dr. rer. physiol. Martina Hahn

Groß $V$ et al. Effectiveness of psychotherapy in adult ADHD: what do patients think? Results of the COMPAS study. J Atten Disord 2017; doi: 10.1177/1087054717720718. [Epub ahead of print] 